\title{
Co-inoculation with Yeast and LAB Under Winery Conditions: Modification of the Aromatic Profile of Merlot Wines
}

\author{
G. Antalick ${ }^{1,2 * \dagger}$, M.C. Perello ${ }^{1,2}$ and G. de Revel ${ }^{1,2}$ \\ (1) University of Bordeaux, ISVV, EA 4577 Enologie, F-33140 Villenave d'Ornon, France \\ (2) INRA, ISVV, USC 1366 Enologie, F-33140 Villenave d'Ornon, France
}

Submitted for publication: February 2013

Accepted for publication: August 2013

Key words: Malolactic fermentation, co-inoculation, Oenococcus oeni, aromatic profile, metabolites

\begin{abstract}
The present work reports the impact of yeast/LAB co-inoculation on the aromatic profile of Merlot wines made in wineries. This study was carried out over two consecutive years on five Merlot wines in Bordeaux and Swiss wineries, using Saccharomyces cerevisiae and Oenococcus oeni starter cultures. Seventy aromatic compounds were quantified and, in addition, the sensory profiles of two wines were determined in order to compare the aromatic notes of the sequential and co-inoculated wines. The influence of the timing of inoculation with lactic acid bacteria (LAB) on the metabolic profile of wines was observed. It confirmed previous work carried out on a micro-scale but, for the first time, the impact of yeast/LAB co-inoculation was significantly demonstrated from a sensory point of view under winery conditions. In particular, the fruity and lactic notes, as well as the markers associated with these descriptors, such as esters and diacetyl, were altered. Co-inoculation does not always favour fruity expression, nor does it reduce the diacetyl content and lactic aroma intensity. All of the trends were observed either in the production and degradation of metabolites, or by the development of an aromatic mask over the short and long term.
\end{abstract}

\section{INTRODUCTION}

The vinification process involves different microbiological processes in which the yeast Saccharomyces cerevisiae, which conducts the alcoholic fermentation (AF), and lactic acid bacteria (LAB), which conducts malolactic fermentation (MLF), play a central role. MLF is carried out mainly by the species Oenococcus oeni and entails an enzymatic decarboxylation of L-malic acid to L-lactic acid and carbon dioxide. It represents an essential step in the improvement of the quality of most red wines, and certainly of white wines (Ribéreau-Gayon et al., 2000).

Malolactic fermentation typically occurs after alcoholic fermentation, either spontaneously as a result of the LAB naturally present in the must, or after inoculation with a starter culture in order to control this step of winemaking (Nielsen et al., 1996). Actually, the timing of the start of MLF depends on several oenological parameters, such as $\mathrm{pH}$, temperature, alcohol content and the sulphur dioxide $\left(\mathrm{SO}_{2}\right)$ concentration, as well as on certain yeast metabolites, such as medium-chain fatty acids (Alexandre et al., 2004) and peptidic fractions (Nehme et al., 2010). Thus, with regard to the oenological conditions, the success of MLF is not always guaranteed and the addition of a starter culture can improve its viability. Overall, two possibilities of LAB inoculation exist: traditional inoculation after alcoholic fermentation (sequential), or simultaneous inoculation in the must with yeast (co-inoculation).

Co-inoculation has several clear benefits compared to the sequential technique. The first advantage is that, by introducing $\mathrm{LAB}$ at the beginning of $\mathrm{AF}$, it helps the bacteria to adapt to the medium better. Secondly, the contents of some compounds that are known to inhibit LAB growth, such as ethanol and $\mathrm{SO}_{2}$, are lower and the medium is richer in nutritive elements during the first hours of AF than at the end. Moreover, it is now well known that co-inoculation reduces the total fermentation time (Rosi et al., 2006; Jussier et al., 2006; Massera et al., 2009; Abrahamse \& Bartowsky, 2012; Knoll et al., 2012; Pan et al., 2011). This reduction limits the risk of spoilage by other microorganisms, such as the Brettanomyces species, which are mainly responsible for 4-ethylphenol production (Jussier et al., 2006; Curtin et al., 2007; Gerbaux et al., 2009). In addition, the thermoregulation time in the tanks can be reduced, thereby decreasing the wineries' energy costs.

Despite all of these advantages, yeast/LAB coinoculation has only been used intermittently because of fear that the quality of the wine will be compromised due to the heterofermentative metabolism of LAB in musts rich in

*Corresponding author: E-mail: gantalick@csu.edu.au

†Present Address: National Wine and Grape Industry Centre, Charles Sturt University, Boorooma Street, Wagga Wagga NSW 2650 Aknowledgements: The authors wish to thank Dr A.C. Silvestri and Prof. S. Hautier of the Ecole d'ingenieurs de Changins, Lallemand Inc., and especially O. Pillet of Laffort Oenologie. Special thanks go to Dr V. Renouf, for carrying out all of the experiments under winery conditions. We also thank the Aquitaine Regional Council and the Bordeaux Wine Council (Conseil Interprofessionnel des Vins de Bordeaux, CIVB), for their financial support 
sugar, which can result in a dramatic increase in acetic acid production (Rasmussen et al., 1995; Edwards et al., 1999). However, recently, numerous studies have shown that the successful completion of MLF induced by co-inoculation has not resulted in adverse increases in volatile acidity (Krieger \& Arnink, 2003; Jussier et al., 2006; Massera et al., 2009; Abrahamse \& Bartowsky, 2011; Knoll et al., 2011; Pan et al., 2011). Thus, the co-inoculation technique recently has been adopted by many more winemakers, particularly in warm climates, where high concentrations of ethanol can strongly inhibit LAB growth (Zapparoli et al., 2009).

Although co-inoculation has been studied well from a fermentation and sanitary point of view (Massera et al., 2009), few studies have focused on the impact of this technique on the aromatic and biochemical profile of wines. Two works have recently shown that the timing of inoculation with LAB in white and red wines could influence the profile of about thirty aromatic yeast-derived compounds such as esters, higher alcohols, fatty acids and varietal compounds such as terpenes (Abrahamse \& Bartowsky, 2011; Knoll et al., 2011). However, in these cases, fermentations were carried out in 1 or $2 \mathrm{~L}$ wine samples under laboratory conditions, which are far from real winery conditions, and no sensory analyses could be performed. From a theoretical point of view, coinoculation is often associated with a decrease in lactic notes due to reduced diacetyl production as the result of carrying out MLF in the presence of active yeast cells (Krieger \& Arnink, 2003). However, this has never been demonstrated experimentally. In contrast, a very recent study has shown that the use of yeast/LAB co-inoculation could lead to increases in diacetyl content (Izquierdo Cañas et al., 2012). It also confirmed the impact of inoculation time with LAB on the metabolite profiles of wines, but additional sensory analyses could not establish any significant changes in wine aroma. A previous work had already shown that yeast/LAB co-inoculation does not have a significant impact on wine aroma, but without quantifying any aromatic compounds (Massera et al., 2009). This previously observed lack of sensory impact of yeast/LAB co-inoculation on wine aroma might reflect the use of micro-scale vinifications. Moreover, the number of yeast/LAB combinations used in all of these studies was limited, and therefore it is more difficult to ascertain the real impact of this oenological practice on wine aroma.

The aim of the present work was to conduct, for the first time, a concurrent sensorial and quantitative study that deals with the impact of yeast/LAB co-inoculation on the aromatic profile of wines under real winery conditions. The study involved five different Merlot wines made in Bordeaux and Swiss wineries with the use of five different yeast/bacteria combinations. The trials could not be replicated due to the experimental limits resulting from the decision to work under winery conditions. However, controls were carried out in order to check the success of yeast and LAB inoculation in the wines. This strategy enabled the experimental conditions to be varied as much as possible in order to best reflect the real impact of co-inoculation on the aromatic and metabolic profiles of Merlot wines. Merlot is one of the most widely planted red cultivars in the world. For each wine, the coinoculation technique was compared to the sequential technique through a quantitative analysis of 70 aromatic compounds. The influence of yeast/LAB co-inoculation on half of these compounds was studied for the first time. Two wines were subjected to a sensory analysis in order to compare the aromatic profiles and to try to establish links with the chemical data.

\section{MATERIALS AND METHODS \\ Wines}

This study was carried out with five Merlot wines from different wineries in Bordeaux (France) and Switzerland and from two vintages (2008 and 2009) (Table 1). The wines were made under winery conditions on five different estates in tanks with volumes greater than $3 \mathrm{hl}$. For each wine, the grapes were crushed following standard protocols and the must obtained was separated into two different tanks, corresponding to the two treatments. Both treatments combined AF performed with Saccharomyces cerevisiae starter cultures and MLF carried out with Oenococcus oeni starter cultures in which LAB were inoculated either during AF (co-inoculation) or after completion of AF (sequential inoculation). In order to vary the experimental conditions as much as possible, five yeast/LAB combinations were tested using commercial strains (one combination per wine). The oenological parameters of the experiments are summarised in Table 1. Only one trial was performed per wine, but controls were carried out to check that the inoculation was successful by taking samples when the malic acid content was decreased by half. All of the strains of LAB managed to colonise the wines in both inoculation techniques (data

TABLE 1

Oenological parameters of the samples.

\begin{tabular}{|c|c|c|c|c|c|c|c|c|c|c|}
\hline Wine & Origin & Year & $\begin{array}{c}\text { Initial } \\
\text { malic } \\
\text { acid } \\
(\mathrm{g} / \mathrm{l}) \\
\end{array}$ & pH & $\begin{array}{c}\text { Alcohol } \\
\text { degree } \\
(\mathrm{v} / \mathrm{v}) \\
\end{array}$ & $\begin{array}{c}\text { Yeast/LAB } \\
\text { combinations }\end{array}$ & $\begin{array}{c}\text { Timing of } \\
\text { bacteria addition }\end{array}$ & $\begin{array}{c}\text { aTotal time of } \\
\text { fermentation in } \\
\text { sequential inoculation } \\
\text { (days) }\end{array}$ & $\begin{array}{c}\text { aTotal time of } \\
\text { fermentation in } \\
\text { co-inoculation } \\
\text { (days) } \\
\end{array}$ & $\begin{array}{l}\text { Timing of MLF } \\
\text { compared to AF } \\
\text { in co-inoculation }\end{array}$ \\
\hline M1 & Switzerland & 2008 & 4 & 3.3 & 12.5 & $\mathrm{U} / \mathrm{F}$ & $24 \mathrm{~h}$ after yeast & 34 & 21 & sequential \\
\hline M2 & Bordeaux & 2009 & 2.1 & 3.6 & 14.0 & $\mathrm{~W} / \mathrm{F}$ & $2 / 3 \mathrm{AF}$ & 54 & 19 & simultaneous \\
\hline M3 & Bordeaux & 2009 & 2.4 & 3.6 & 12.3 & $\mathrm{X} / \mathrm{B}$ & $24 \mathrm{~h}$ after yeast & 36 & 18 & simultaneous \\
\hline M4 & Bordeaux & 2009 & 1.6 & 3.8 & 14.2 & $\mathrm{Y} / \mathrm{B}$ & $24 \mathrm{~h}$ after yeast & 44 & 14 & simultaneous \\
\hline M5 & Bordeaux & 2009 & 2.1 & 3.7 & 13.5 & $\mathrm{Z} / \mathrm{I}$ & $24 \mathrm{~h}$ after yeast & 78 & 28 & sequential \\
\hline
\end{tabular}

atotal time fermentation corresponds to the addition of AF and MLF time in days. 
not shown). Alcoholic fermentation was considered to be finished when the sugar content reached $2 \mathrm{~g} / \mathrm{L}$, whereas malolactic fermentation was stopped when the malic acid concentration dropped below $0.2 \mathrm{~g} / \mathrm{L}$. At the end of MLF, about $50 \mathrm{mg} / \mathrm{L}$ of $\mathrm{K}_{2} \mathrm{SO}_{2}$ was added and the wines were racked and bottled. Wines M1 and M2 were kept at $12^{\circ} \mathrm{C}$ for four months to stabilise them before sampling; a sample of each wine was frozen for subsequent chemical analysis and another sample was prepared for a sensory analysis that was carried out at the same time. For wines M3 to M5, which were not subjected to sensory analysis, samples were taken two weeks after bottling to be frozen for subsequent chemical analysis.

\section{Standard chemical analyses}

The standard chemical parameters of the wines were determined according to the methods outlined by the International Organisation of Vine and Wine (OIV) (data not shown).

\section{Volatile compound analyses}

Each treatment (sequential and co-inoculation) was simultaneously analysed after thawing, which did not have an impact on the aroma compound content of the racked wine. Seventy molecules were analysed using seven different methods developed and validated in our laboratory. The measurements were duplicated for all of the analyses and the values displayed corresponded to the mean of these duplicates. However, standard deviations are not provided because the repeatability was only analytical and not microbiological.

\section{Diacetyl (liquid-liquid extraction after derivatisation and GC-MS analysis)}

Diacetyl contents were measured using the method developed by De Revel et al. (2000).

\section{Acetoin and butanediols (direct injection and GC/FID analysis)}

Acetoin, D-butan-2,3-diol and L-butan-2,3-diol were quantified with the method developed by De Revel (1992). According to this method, $1 \mathrm{~mL}$ of wine was spiked with 50 $\mu \mathrm{L}$ of internal standard solution and diluted with $2 \mathrm{~mL}$ of methanol. The vials were filled with this solution for direct injection into a gas chromatograph Agilent 6890N coupled to a flame ionisation detector (FID). The column was a FFAP type (BP 21, $50 \mathrm{~m}$ x $0.25 \mathrm{~mm}$ x $0.2 \mu \mathrm{m}$, SGE). Quantification was performed using a calibration curve obtained from $12 \%$ hydroalcoholic solution. Butan-1,4-diol at $1 \mathrm{~g} / \mathrm{L}$ in $40 \%$ hydroalcoholic solution was used as an internal standard.

\section{Volatile sulphur compounds (HS-GC/FPD)}

Dimethyl sulphide (DMS) and hydrogen sulphide $\left(\mathrm{H}_{2} \mathrm{~S}\right)$ were analysed using the method proposed and validated by Anocibar-Beloqui et al. (1996).

\section{C13-norisoprenoids and lactones (SBSE-GC/MS)}

The method developed and validated by Antalick (2010) was used to quantify four C13-norisoprenoids ( $\beta$-damascenone, $\beta$-damascone, $\beta$-ionone and $\alpha$-ionone) and six lactones $(\gamma$-octalactone, $\gamma$-nonalactone, $\gamma$-decalactone, $\gamma$-undecalactone, $\gamma$-dodecalactone and $\delta$-decalactone).

\section{A-polar esters (HS-SPME-GC/MS)}

The method developed and validated by Antalick et al. (2010) was used to quantify 32 a-polar esters: fatty acid ethyl esters, higher alcohol acetates, branched acid ethyl esters, isoamyl esters, methyl esters, ethyl cinnamates and minor esters.

\section{Ethyl acetate, ethanal, methanol and higher alcohol (direct injection and GC/FID analysis)}

Ethyl acetate, total ethanal and higher alcohols were quantified using a modified version of the official OIV method (OIV-MA-AS315-02A). According to this method, $5 \mathrm{~mL}$ of wine was spiked with $50 \mu \mathrm{L}$ of internal standard solution. The vials were filled with this solution for direct injection into a gas chromatograph Agilent $6890 \mathrm{~N}$ coupled to a flame ionisation detector (FID). The column was a CP-Wax $57 \mathrm{CB}$ (50 m x $0.25 \mathrm{~mm} \times 0.2 \mu \mathrm{m}$, Varian). Quantification was performed using a calibration curve obtained from $12 \%$ hydroalcoholic solution. 4-Methylpentan-2-ol at 10 $\mathrm{g} / \mathrm{L}$ in $50 \%$ hydroalcoholic solution was used as an internal standard.

Volatile fatty acids (liquid-liquid extraction and GC/FID) Hexanoic, octanoic, decanoic and dodecanoic acids were quantified by the method previously developed by Bertrand (1981). According to this method, $50 \mathrm{~mL}$ of wine previously spiked with $200 \mu \mathrm{L}$ of internal standard solution was successively extracted with $4 \mathrm{ml}$ and twice with $2 \mathrm{ml}$ of an diethyl ether/isohexane mix $(1: 1, \mathrm{v} / \mathrm{v})$. The organic phases were collected and injected into a gas chromatograph HP5890 coupled to a flame ionisation detector (FID). The column was an FFAP type (BP 21, $50 \mathrm{~m}$ x $0.25 \mathrm{~mm} \times 0.2$ $\mu \mathrm{m}$, SGE). Measurements were carried out following the protocol described by Bertrand (1981). Quantification was performed with calibration curves obtained from red wines. Octan-3-ol at $20 \mathrm{mg} / \mathrm{L}$ in alcoholic solution was used as an internal standard.

\section{Additional volatile compounds (liquid-liquid extraction and $G C / M S$ analysis)}

The method developed and validated by Antalick (2010) was used to quantify seven polar esters (ethyl lactate, ethyl succinates and hydroxylated ethyl esters), three branched acids (isobutyric acid, isovaleric acid and 2-methylbutyric acid) and linalool.

\section{Sensory analysis}

The sensory analyses were carried out by orthonasal evaluation, using odour comparison profiles (Martin \& de Revel, 1999) in order to compare the aromatic profiles of wines made with the sequential and co-inoculation techniques and from the same two malolactic starter cultures. A list of four descriptive terms was previously designated at the laboratory; simple descriptors were chosen in order to simplify the evaluation and to keep the panel's attention. The chosen terms were consistent with a fruity aroma (fruity), MLF (lactic) and an overall aroma potentially impacting on 
the fruity aroma (vegetal and smoked/toasted). The panellists evaluated the intensity of the four aromatic attributes on a scale from 1 to 7 . All of the panellists were members of the Faculty of Oenology's sensory panel (University of Bordeaux) and were used to tasting wine. The number of panellists $(n)$ varied between the tests $(n=13$ to 16$)$ depending on personal availability, with women comprising $39 \%$ to $54 \%$ of the panels.

All of the tasting sessions took place in a dedicated air-conditioned room $\left(20^{\circ} \mathrm{C}\right)$ equipped with individual booths. The samples $(50 \mathrm{~mL})$ were presented under "normal daylight" illumination in normalised dark glasses (ISO 3591, 1977) identified with random three-digit codes.

Two Merlot wines (M1 and M2) were subjected to a sensory analysis.

\section{Statistical analysis}

For the sensory analysis, a two-factor analysis of variance (product and judge) and student t-tests were carried out in order to identify significant differences in the intensity of the attributes noted by the panel members.

The analysis of variance and student t-tests were carried out using Excel software (Microsoft Corporation, Redmond, USA).

\section{RESULTS}

\section{Malolactic fermentation}

Five combinations of yeast/bacteria starter cultures (five yeasts and three bacteria) were tested in five different Merlot wines made under winery conditions. Some of the analytical and technical parameters of the wines are shown in Table 1. MLF succeeded in every case, regardless of the inoculation technique. A malolactic starter was added to the wines 24 hours after yeast inoculation, except to wine $\mathrm{M} 2$, in which the starter was added two thirds of the way through the alcoholic fermentation. The wines displayed 1.6- to 2.8-fold decreases in the total fermentation time in the co-inoculation technique compared to the sequential technique. In wines M2, M3 and M4, made by co-inoculation, MLF occurred during AF, whereas in wines M1 and M5, MLF induced by co-inoculation occurred after alcoholic fermentation.

\section{Influence of yeast/LAB co-inoculation on wine aromatic markers}

Seventy aromatic compounds, including esters, higher alcohols, acids, sulphur-containing compounds, diacetyl, butan-2,3-diols, acetoin, C13-norisoprenoids, lactones and linalool, were quantified using suitable analytical methods that were previously developed and validated in our laboratory. Tables 2, 3 and 4 list the concentrations of the aromatic compounds determined in the wines after MLF was induced by yeast/LAB co-inoculation and sequential inoculation. These measurements show that the timing of inoculation with LAB had an impact on the metabolic profile of the wines.

\section{Esters}

Two groups of esters could be identified: major esters and odorant esters. Major esters corresponding to ethyl acetate, ethyl lactate, and ethyl and diethyl succinate were quantitatively present at high levels in the wines $(\mathrm{mg} / \mathrm{L})$. The other quantified esters were considered to be odorant esters because they had a much higher impact on wine aroma, despite having lower concentrations $(\mu \mathrm{g} / \mathrm{L})$. Table 2 shows the ester concentrations measured after MLF in the coinoculated wines and those made with sequential inoculation.

With regard to the major ester group, ethyl acetate displays the lowest concentration variations overall as a result of the timing of inoculation. Many different kinds of variations were observed, even though co-inoculation tended to increase the levels of ethyl acetate (Table 2). This trend was also observed with succinates, mainly with diethyl succinate. Overall, the influence of the timing of inoculation was larger for succinate than for the other major esters. Ethyl lactate was also affected by co-inoculation, but there were no obvious trends (Table 2).

The timing of LAB inoculation also influenced the composition of odorant esters, with variations measured from 150 to $1200 \mu \mathrm{g} / \mathrm{L}$. Many different kinds of variations were observed with co-inoculation, which led to higher levels of odorant esters in three wines (M1, M3 and M4), whereas the ester content was higher after sequential inoculation in two cases (M2 and M5). The variations depended on the group of esters considered.

Co-inoculation favoured higher fatty acid ethyl ester contents in three wines (M1, M3, M4) and led to decreases in the levels in the two remaining wines (M2, M5). Overall, the variations in the entire group of these esters followed the same trend in the wines analysed, except ethyl propanoate, which seemed to follow the same trend as the branched acid ethyl esters, with a concentration increase observed in only one wine (M1). Two wines displayed higher levels of higher alcohol acetates in the co-inoculation technique (M1 and M4). Moreover, unlike fatty acid ethyl esters, no trend could be emphasised in the impact of co-inoculation on individual higher alcohol acetate contents. Methyl and isoamyl esters, which are part of the minor ester group, followed the same variations as the fatty acid ethyl esters.

\section{Other fermentation-derived compounds}

Other compounds derived from the fermentation process other than esters were quantified, including diacetyl, acetoin, ethanal, alcohols, acids and sulphur-containing compounds (Table 3).

The timing of inoculation strongly affected the diacetyl content, which increased in four out of five cases (Table 3). All of the compounds derived from diacetyl reduction (acetoin and butan-2,3-diols) followed the same variations. As with diacetyl, co-inoculation led to an overall higher contents of acetoin and butan-2,3-diols; however, the measured relative variations were more limited (max. 20\%). Ethanal was also affected by co-inoculation, without any specific trend observed under our conditions (Table 3).

Higher alcohols and methanol were not influenced greatly by the LAB inoculation technique (except for wine M2). Conversely, the influence was much more significant on the acid composition than on the alcohol composition, and many different kinds of variations were observed. Two groups can be distinguished in which all of the esters followed the same trends: branched acids and fatty acids. 
TABLE 2

Comparison of the ester concentrations $(\mu \mathrm{g} / \mathrm{L})$ between wines made by yeast/LAB co-inoculation (Co-in.) and sequential inoculation (Seq.in.).

\begin{tabular}{|c|c|c|c|c|c|c|c|c|c|c|}
\hline \multirow{2}{*}{ Compounds } & \multicolumn{2}{|c|}{ M1 } & \multicolumn{2}{|c|}{ M2 } & \multicolumn{2}{|c|}{ M3 } & \multicolumn{2}{|c|}{ M4 } & \multicolumn{2}{|c|}{ M5 } \\
\hline & Co-in & Seq. in. & Co-in & Seq. in. & Co-in & Seq. in. & Co-in & Seq. in. & Co-in & Seq. in. \\
\hline \multicolumn{11}{|l|}{ Fatty acid ethyl esters } \\
\hline Ethyl propanoate & 54 & 42 & 56 & 60 & 86 & 87 & 103 & 119 & 99 & 105 \\
\hline Ethyl butyrate & 187 & 180 & 247 & 370 & 217 & 187 & 142 & 127 & 174 & 221 \\
\hline Ethyl valerate & 0.42 & 0.32 & 0.64 & 0.45 & $\mathrm{nq}^{*}$ & $\mathrm{nq}$ & 0.60 & 0.76 & 0.74 & 0.91 \\
\hline Ethyl hexanoate & 309 & 277 & 454 & 688 & 543 & 495 & 265 & 243 & 327 & 422 \\
\hline Ethyl heptanoate & 0.52 & 0.50 & 0.58 & 0.64 & 0.60 & 0.55 & 0.43 & 0.52 & 0.74 & 0.81 \\
\hline Ethyl octanoate & 457 & 387 & 581 & 820 & 680 & 567 & 376 & 327 & 415 & 537 \\
\hline Ethyl nonanoate & 0.64 & 0.73 & 0.53 & 0.53 & 0.92 & 0.76 & 0.76 & 0.53 & 0.68 & 0.69 \\
\hline Ethyl decanoate & 113 & 51 & 207 & 314 & 203 & 184 & 164 & 117 & 137 & 178 \\
\hline Ethyl dodecanoate & 9.95 & 2.28 & 8.37 & 10.43 & 6.14 & 5.82 & 4.50 & 6.13 & 4.35 & 7.74 \\
\hline Sum & 1132 & 940 & 1554 & 2264 & 1738 & 1530 & 1057 & 942 & 1158 & 1473 \\
\hline \multicolumn{11}{|l|}{ Higher alcohol acetates } \\
\hline Propyl acetate & 20.5 & 15.3 & 10.3 & 10.0 & 13.4 & 13.1 & 8.0 & 6.7 & 8.3 & 9.2 \\
\hline Isobutyl acetate & 104 & 89 & 45 & 63 & 60 & 81 & 27 & 37 & 50 & 74 \\
\hline Butyl acetate & 1.6 & 0.9 & 1.0 & 0.7 & 1.1 & 0.8 & nq & $\mathrm{nq}$ & $\mathrm{nq}$ & $\mathrm{nq}$ \\
\hline Isoamyl acetate & 1645 & 1467 & 663 & 846 & 1724 & 1728 & 593 & 436 & 345 & 370 \\
\hline Hexyl acetate & 10.1 & 6.9 & 2.33 & 1.82 & 10.30 & 14.86 & 0.85 & 0.58 & 0.73 & 0.85 \\
\hline Octyl acetate & 0.16 & 0.05 & $\mathrm{nq}$ & $\mathrm{nq}$ & 0.07 & 0.07 & 0.02 & 0.02 & 0.41 & 0.43 \\
\hline Phenylethyl acetate & 95 & 66 & 35 & 38 & 172 & 202 & 49 & 47 & 31 & 35 \\
\hline Sum & 1876 & 1646 & 757 & 960 & 1922 & 1959 & 684 & 533 & 440 & 494 \\
\hline \multicolumn{11}{|l|}{ Branched acid ethyl esters } \\
\hline Ethyl isobutyrate & 52 & 51 & 35 & 44 & 26 & 31 & 57 & 73 & 39 & 44 \\
\hline Ethyl 2-methylbutyrate & 6.8 & 5.7 & 6.1 & 6.5 & 6.7 & 7.8 & 9.5 & 15.1 & 8.4 & 9.5 \\
\hline Ethyl isovalerate & 10.9 & 11.0 & 12.2 & 14.5 & 10.6 & 14.1 & 16.9 & 24.5 & 13.8 & 16.8 \\
\hline Ethyl phenylacetate & 2.39 & 1.51 & 2.29 & 2.35 & 5.26 & 6.77 & 4.40 & 6.23 & 3.88 & 4.69 \\
\hline Sum & 72 & 69 & 56 & 67 & 49 & 60 & 87 & 118 & 65 & 75 \\
\hline \multicolumn{11}{|l|}{ Cinnamates } \\
\hline Ethyl cinnamate & 1.31 & 1.07 & 0.51 & 0.57 & 1.21 & 1.00 & 0.90 & 1.08 & 1.27 & 0.87 \\
\hline Ethyl dihydrocinnamate & 1.61 & 1.08 & 0.36 & 0.47 & 1.08 & 1.02 & 1.21 & 1.38 & 0.69 & 0.85 \\
\hline Sum & 2.92 & 2.15 & 0.87 & 1.04 & 2.29 & 2.02 & 2.11 & 2.47 & 1.95 & 1.72 \\
\hline \multicolumn{11}{|l|}{ Miscellaneous esters } \\
\hline Methyl butyrate & 0.5 & 0.4 & 1.8 & 2.5 & nq & nq & nq & nq & nq & nq \\
\hline Methyl hexanoate & 0.91 & 0.80 & 2.26 & 3.16 & 1.32 & 1.14 & 1.15 & 0.97 & 1.11 & 1.29 \\
\hline Methyl octanoate & 1.06 & 0.86 & 2.30 & 3.67 & 1.36 & 1.15 & 1.05 & 0.93 & 1.18 & 1.44 \\
\hline Methyl decanoate & 0.19 & 0.07 & 0.56 & 0.89 & 0.32 & 0.26 & 0.33 & 0.25 & 0.29 & 0.35 \\
\hline Isoamyl butyrate & 0.47 & 0.45 & 0.55 & 0.90 & 0.61 & 0.55 & 0.32 & 0.32 & 0.42 & 0.57 \\
\hline Isoamyl hexanoate & 1.02 & 1.03 & 1.14 & 2.08 & 2.08 & 1.78 & 0.70 & 0.65 & 0.85 & 1.16 \\
\hline Isoamyl octanoate & 1.68 & 1.66 & 1.74 & 3.37 & 2.77 & 2.59 & 1.29 & 1.19 & 1.25 & 1.80 \\
\hline Ethyl 3-hydroxybutyrate & 233 & 141 & 341 & 561 & 438 & 428 & 238 & 316 & 223 & 256 \\
\hline Ethyl trans-2-hexenoate & 0.79 & 0.59 & 2.80 & 2.31 & 1.66 & 2.23 & 1.07 & 0.73 & 1.49 & 1.75 \\
\hline Isobutyl hexanoate & 0.17 & 0.20 & 0.13 & 0.34 & 0.13 & 0.12 & 0.07 & 0.06 & 0.10 & 0.13 \\
\hline Methyl geranate & 0.16 & 0.07 & 0.17 & 0.21 & 0.14 & 0.14 & 0.70 & 0.65 & 0.28 & 0.33 \\
\hline Sum of odorant esters & 3324 & 2804 & 2722 & 3873 & 4226 & 4078 & 2078 & 1921 & 1900 & 2316 \\
\hline \multicolumn{11}{|l|}{ Major esters } \\
\hline Ethyl acetate & 39000 & 37000 & 63360 & 58200 & 51000 & 30000 & 5750 & 8150 & 58840 & 47760 \\
\hline Ethyl lactate & 113000 & 54000 & 33000 & 33400 & 38600 & 48350 & 48550 & 54200 & 35340 & 33700 \\
\hline Diethyl succinate & 1321 & 585 & 357 & 782 & 7335 & 2918 & 5863 & 3794 & 3314 & 1603 \\
\hline Monoethyl succinate & 24000 & 9180 & 31060 & 93165 & 267000 & 286000 & 2880000 & 309000 & 278100 & 112200 \\
\hline
\end{tabular}

*nq: non-quantifiable (< LOQ). 
TABLE 3

Comparison of the fermentation-derived compound concentrations $(\mathrm{mg} / \mathrm{L})$ between wines made by yeast/LAB co-inoculation (Co-in.) and sequential inoculation (Seq.in.).

\begin{tabular}{|c|c|c|c|c|c|c|c|c|c|c|}
\hline \multirow{2}{*}{ Compounds } & \multicolumn{2}{|c|}{ M1 } & \multicolumn{2}{|c|}{ M2 } & \multicolumn{2}{|c|}{ M3 } & \multicolumn{2}{|c|}{ M4 } & \multicolumn{2}{|c|}{ M5 } \\
\hline & Co-in & Seq. in. & Co-in & Seq. in. & Co-in & Seq. in. & Co-in & Seq. in. & Co-in & Seq. in. \\
\hline Diacetyl & 4.6 & 9.2 & 4.0 & 3.2 & 2.1 & 1.8 & 4.6 & 1.6 & 1.9 & 1.1 \\
\hline \multicolumn{11}{|l|}{$\begin{array}{l}\text { Compounds derived from } \\
\text { diacetyl reduction }\end{array}$} \\
\hline Acetoin & 14.1 & 10.4 & 25.5 & 23.1 & 13.2 & 14.4 & 7.9 & 11.9 & 12.7 & 9.3 \\
\hline Butan-2,3-diol & 175 & 154 & 72 & 70 & 109 & 99 & 104 & 129 & 101 & 99 \\
\hline Butan-2,3-diol & 42 & 42 & 24 & 19 & 37 & 37 & 40 & 51 & 48 & 47 \\
\hline Sum & 231 & 206 & 122 & 112 & 160 & 150 & 153 & 192 & 162 & 155 \\
\hline \multicolumn{11}{|l|}{ Higher alcohol } \\
\hline Propanol & 27 & 26 & 14 & 13.5 & 12.9 & 11.9 & 9.8 & 10.4 & 15 & 15.4 \\
\hline Isobutanol & 80 & 86 & 44 & 71 & 31 & 31 & 32 & 29 & 40 & 38 \\
\hline Butanol & 1.1 & 0.7 & 1.0 & 0.8 & 1.0 & 1.0 & 0.6 & 0.8 & 0.6 & 0.7 \\
\hline 2-methylbutanol & 76 & 79 & 44 & 49 & 59 & 61 & 44 & 47 & 46 & 47 \\
\hline 3-methylbutanol & 255 & 271 & 163 & 194 & 194 & 195 & 159 & 160 & 165 & 171 \\
\hline Hexanol & $-\mathrm{b}$ & - & 0.61 & 0.43 & 0.68 & 0.82 & 0.35 & 0.28 & 0.30 & 0.33 \\
\hline Sum & 440 & 464 & 267 & 330 & 298 & 300 & 246 & 247 & 267 & 273 \\
\hline \multicolumn{11}{|l|}{ Acids } \\
\hline Isobutyric acid & 1.23 & 1.22 & 1.48 & 1.61 & 1.23 & 1.23 & 3.63 & 3.04 & 1.25 & 1.56 \\
\hline 2-methylbutyric acid & 0.63 & 0.40 & 0.86 & 0.86 & 1.09 & 1.17 & 1.36 & 1.35 & 0.98 & 1.07 \\
\hline Isovaleric acid & 0.24 & 0.12 & 0.21 & 0.22 & 0.48 & 0.86 & 0.78 & 0.62 & 0.44 & 0.36 \\
\hline Hexanoic acid & - & - & 2.55 & 3.81 & 2.88 & 2.36 & 1.62 & 1.44 & 1.82 & 1.95 \\
\hline Octanoic acid & - & - & 2.21 & 3.62 & 2.15 & 1.71 & 1.36 & 1.27 & 1.34 & 1.42 \\
\hline Decanoic acid & - & - & 0.49 & 0.73 & 0.45 & 0.37 & 0.39 & 0.33 & 0.29 & 0.33 \\
\hline Dodecanoic acid & - & - & 0.02 & 0.02 & 0.02 & 0.01 & 0.01 & 0.01 & 0.01 & 0.01 \\
\hline Sum & - & - & 7.81 & 10.87 & 8.30 & 7.71 & 9.15 & 8.06 & 6.13 & 6.71 \\
\hline \multicolumn{11}{|c|}{ Sulphur-containing compounds } \\
\hline Hydrogen sulphide ${ }^{a}$ & 1.90 & 1.60 & 2.40 & 2.43 & 3.90 & 3.17 & 2.37 & 2.63 & 2.10 & 2.33 \\
\hline Dimethyl sulphide ${ }^{a}$ & 18.60 & 15.50 & 4.47 & 2.90 & 2.53 & 2.70 & 2.03 & 2.70 & 3.30 & 3.60 \\
\hline \multicolumn{11}{|c|}{ Miscellaneous compounds } \\
\hline Methanol & 132 & 122 & 344 & 326 & 116 & 111 & 162 & 176 & 209 & 187 \\
\hline Ethanal & 4.6 & 3.2 & 29.1 & 14.5 & 6.1 & 8.1 & 14.4 & 18.6 & 14.5 & 10.2 \\
\hline
\end{tabular}

a $\mu \mathrm{g} / \mathrm{L} ;{ }^{\text {b }}$, no data

TABLE 4

Comparison of the varietal compound concentrations $(\mu \mathrm{g} / \mathrm{L})$ between wines made by yeast/LAB co-inoculation (Co-in.) and sequential inoculation (Seq.in.).

\begin{tabular}{|c|c|c|c|c|c|c|c|c|c|c|}
\hline \multirow{2}{*}{ Compounds } & \multicolumn{2}{|c|}{ M1 } & \multicolumn{2}{|c|}{ M2 } & \multicolumn{2}{|c|}{ M3 } & \multicolumn{2}{|c|}{ M4 } & \multicolumn{2}{|c|}{ M5 } \\
\hline & Co-in & Seq. in. & Co-in & Seq. in. & Co-in & Seq. in. & Co-in & Seq. in. & Co-in & Seq. in. \\
\hline \multicolumn{11}{|l|}{ C13-norisoprenoids } \\
\hline$\beta$-damascone & 0.03 & 0.02 & 0.01 & 0.01 & 0.07 & 0.06 & 0.04 & 0.04 & 0.03 & 0.02 \\
\hline$\beta$-damascenone & 2.56 & 2.36 & 2.51 & 2.47 & 3.63 & 4.06 & 2.16 & 1.74 & 2.05 & 2.14 \\
\hline$\alpha$-ionone & 0.10 & 0.12 & 0.17 & 0.10 & 0.21 & 0.18 & 0.14 & 0.10 & 0.28 & 0.10 \\
\hline$\beta$-ionone & 0.07 & 0.06 & 0.10 & 0.08 & 0.12 & 0.11 & 0.10 & 0.07 & 0.12 & 0.12 \\
\hline Sum & 2.76 & 2.56 & 2.79 & 2.66 & 4.03 & 4.41 & 2.43 & 1.95 & 2.47 & 2.37 \\
\hline \multicolumn{11}{|l|}{ Lactones } \\
\hline$\gamma$-octalactone & 0.42 & 0.62 & $\mathrm{nq}$ & nq & 0.99 & 0.93 & 0.63 & 0.82 & 2.11 & 1.53 \\
\hline$\gamma$-nonalactone & 4.12 & 3.18 & 3.61 & 3.25 & 7.25 & 6.40 & 4.12 & 4.50 & 6.53 & 6.46 \\
\hline$\gamma$-decalactone & 0.51 & 0.43 & 0.39 & 0.37 & 0.57 & 0.51 & 0.76 & 0.70 & 0.65 & 0.53 \\
\hline$\gamma$-undecalactone & 0.06 & 0.05 & 0.04 & 0.04 & 0.14 & 0.11 & 0.16 & 0.25 & 0.10 & 0.08 \\
\hline$\gamma$-dodecalactone & 0.10 & 0.08 & 0.03 & 0.04 & 0.08 & 0.14 & 0.12 & 0.13 & 0.11 & 0.11 \\
\hline$\delta$-decalactone & 2.14 & 1.99 & 1.33 & 1.41 & 2.46 & 2.18 & 2.34 & 1.99 & 3.29 & 3.15 \\
\hline Sum & 7.36 & 6.36 & 5.40 & 5.11 & 11.49 & 10.27 & 8.12 & 8.38 & 12.80 & 11.86 \\
\hline \multicolumn{11}{|l|}{ Terpenols } \\
\hline Linalool & 8.4 & 2.9 & 3.7 & 4.6 & 4.2 & 6.0 & 6.6 & 8.1 & 4.9 & 5.6 \\
\hline
\end{tabular}

${ }^{a}$ nq: non-quantifiable (< LOQ). 
$\mathrm{M} 1(\mathrm{n}=13)$

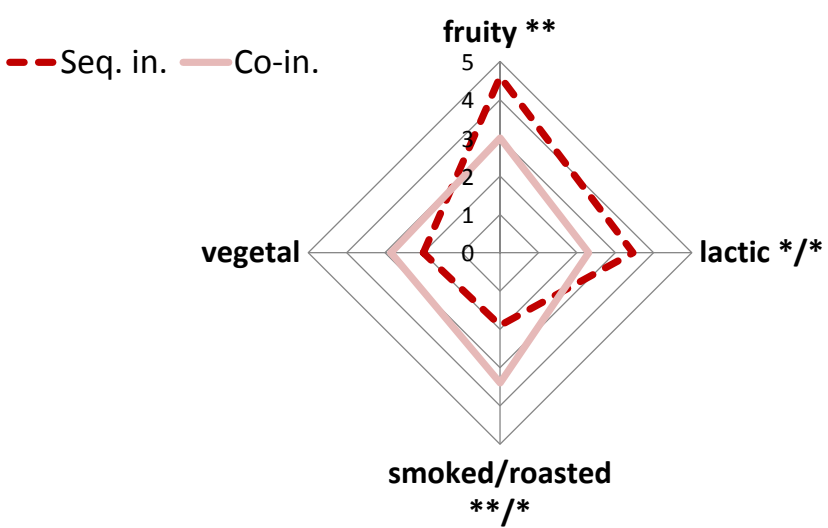

$M 2(n=16)$

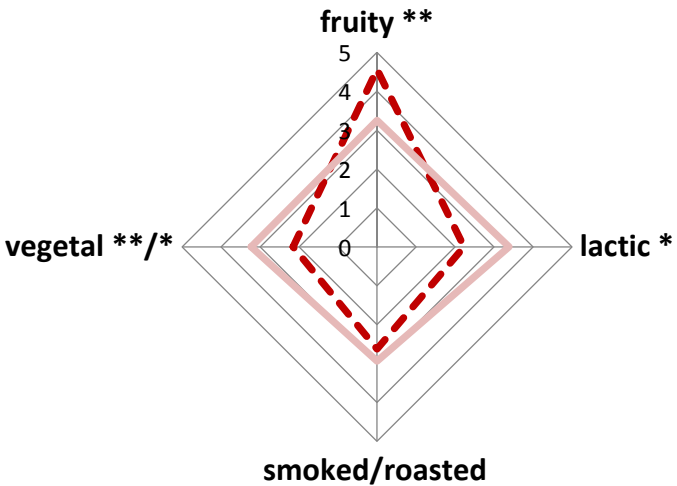

smoked/roasted

FIGURE 1

Odour comparison profiles performed on means of sensory descriptor values in the analysis of wines made by yeast/LAB co-inoculation (Co-in.) and sequential inoculation (Seq.in.). Treatments with significant differences are indicated with stars (product effect/judge effect). $\left(^{*}\right)$, significant at $\mathrm{p}<0.05,(* *)$, significant at $\mathrm{p}<0.01, \mathrm{n}$ indicates the number of panellists for each test.

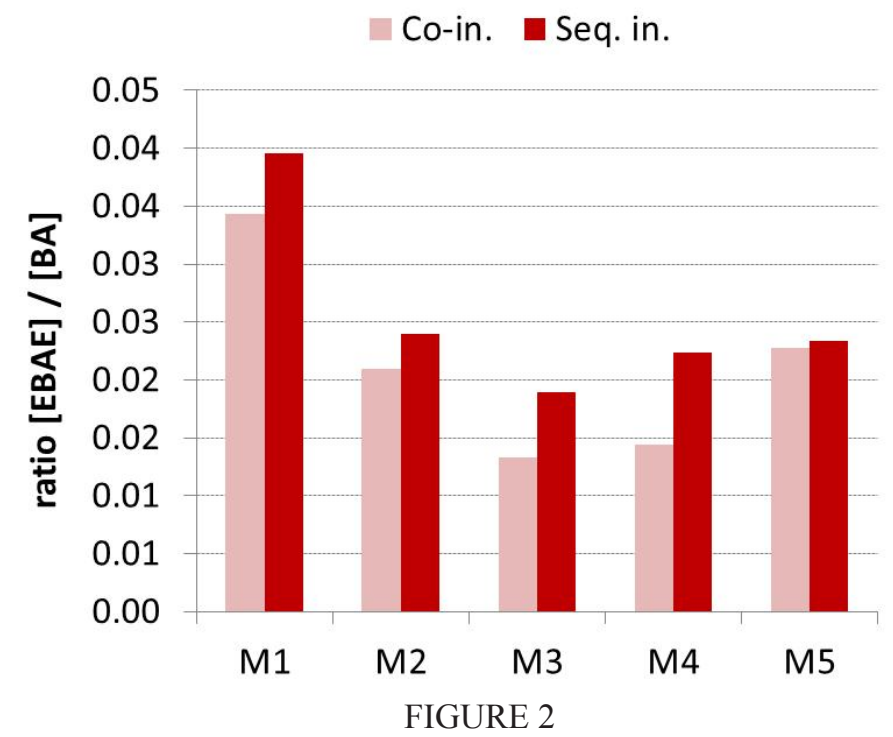

Comparison of ethyl branched acids esters/branched acid concentrations ratios (EBAE/BA) between wines made by yeast/LAB co-inoculation (Co-in.) and sequential inoculation (Seq.in.).

Branched acids were not as affected as fatty acids, with respective relative variations from $-15 \%$ to $+15 \%$ for the first group, and from $-50 \%$ to $+20 \%$ for the second group (Table 3).

Finally, the levels of sulphur-containing compounds, such as hydrogen sulphide and dimethyl sulphide, were slightly affected overall by the timing of inoculation, without any clear trend in the variations.

\section{Varietal compounds}

Overall, co-inoculation with LAB decreased the level of linalool (four out of five wines) (Table 4). However, the levels of variation remained moderate. The levels of C13norisoprenoids were slightly influenced by the inoculation technique. However, $\alpha$-ionone was clearly affected more than the other C13-norisoprenoids, with a clear trend of higher contents in the co-inoculation technique (four out of five cases) (Table 4). For $\beta$-damascenone, $\beta$-damascone and $\beta$-ionone, the differences between the two inoculation techniques were lower or non-existent, and no trends were noted. The variations measured for the lactones were also low, but a clear trend of increases in the lactone content was observed in the co-inoculation technique (four out of five wines) (Table 4).

Influence of yeast/LAB co-inoculation on the aromatic profile of red wines

For technical reasons (volume of samples), only two of the five wines studied in this work were subjected to a sensory analysis (M1 and M2) (Fig. 1). The first result, observed by comparing the profiles, is the significant impact of the timing of inoculation with LAB on the aromatic profile of red wines. All four the descriptors evaluated by the panel were affected. The fruity aroma seemed to be the aromatic note that was 
affected the most, and co-inoculation decreased its intensity. The panel had a harder time judging the other descriptors (judge effect). However, the lactic notes were significantly altered by the method of LAB inoculation in both wines. Coinoculation resulted in a decrease in lactic note intensity in wine M1, whereas it enhanced it in wine M2. The smoked/ toasted and vegetal descriptors were affected less. However, some significant differences were also observed for these notes. Moreover, no off-flavour due to volatile acidity was perceived in the studied wines, irrespective of the inoculation technique used.

\section{DISCUSSION}

One of the main known advantages of yeast/LAB coinoculation is that it significantly reduces the total fermentation time. The present study confirmed this data on a winery scale, and is in agreement with previous work carried out on a laboratory scale and with empirical observations by winemakers (Rosi et al., 2006; Massera et al., 2009). Even though co-inoculation results in a significant reduction in fermentation time, this method does not always lead to simultaneous fermentation. The inoculation of wine with bacteria at the beginning of AF does not guarantee that MLF and AF will be performed simultaneously. After coinoculation, MLF can also be carried out after AF, but even in this case the total fermentation time is reduced, probably because the bacteria that have been present in the medium since the beginning of AF are better adapted to the medium.

Regardless of the timing of MLF, the significant impact of yeast/LAB co-inoculation on the aromatic profile of wine compared to traditional sequential inoculation with LAB was clearly shown. Recent studies have shown the modification of the biochemical profile of wine generated by different timings of inoculation with LAB (Abrahamse \& Bartowsky, 2011; Knoll et al., 2011; Izquierdo Cañas et al., 2012). However, this work showed for the first time the significant impact of co-inoculation on the sensory profile of wines under winery conditions. The few studies that have already focused on this topic were carried out using small volumes (microvinification) (Massera et al., 2009; Mendoza et al., 2011, Izquierdo Cañas et al., 2012). The use of such volumes is very convenient for the consistent study of the fermentation processes from a microbiological and biochemical point of view. However, the conditions are different from those in a winery, and microvinification often generates off-flavours (oxidation, reduction) that disturb the sensory evaluation of the wine. Thus, in the present study, the metabolic changes measured were in accordance with the sensory changes perceived by the panellists.

The fruity and lactic aromas were the descriptors affected the most, but contrary to empirical ideas and predictions made in previous studies, yeast/LAB co-inoculation does not always favour the fruity expression by decreasing the lactic notes linked to diacetyl (Krieger \& Arnink, 2003). Although our study shows that this phenomenon is actually possible (wine M1), co-inoculation increased the diacetyl content in most of the cases, and the lactic aroma was perceived as being more intense after co-inoculation in wine M2. Izquierdo Cañas et al. (2012) have also shown that coinoculation could lead to an increase in the diacetyl level, although without any sensory impact.

Moreover, some authors have suggested that yeast/ LAB co-inoculation could enhance the fruity aroma, thereby increasing the level of esters, which play a central role in the fruity aroma perception in wine (Escudero et al., 2007; Pineau et al., 2009; Abrahamse \& Bartowsky, 2011; Knoll et al., 2011). In contrast, the panellists in our study perceived the two wines made by co-inoculation to be significantly less fruity $(\mathrm{p}<0.01)$. However, this does not mean that yeast/LAB co-inoculation systematically degrades the fruity aroma, as some of our previous work showed an intensification of the fruity aroma after using this technique in a Pinot noir wine (Antalick, 2010). Furthermore, the increase in the content of the fruity markers does not always guarantee the enhancement of a fruity aroma, because other aromatic changes can mask the fruity notes. This was the case in wine M1, for which co-inoculation led to the development of a smoked/toasted note that clearly masked the fruity aroma, especially since the levels of esters were higher than in wines made by sequential inoculation. Coinoculation can also result in decreases in the ester content, associated with a reduction in the intensity of the fruity aroma (M2). Moreover, co-inoculation tends to reduce the levels of branched acid ethyl esters. However, branched acid ethyl esters were synthesised substantially during wine ageing by esterification with ethanol of the corresponding branched acids (Diaz-Maroto et al., 2005). The rates and yields of these esterifications depend strongly on the ester/ acid ratio according to the law of mass action. The reduction of the content of the ethyl-branched acid esters observed in co-inoculation tends to decrease these ratios (Fig. 2). It favours the late synthesis of ethyl-branched acid esters, and therefore the potential development of fruity notes during wine ageing. Thus, even though co-inoculation can actually enhance the fruity aroma, either by lactic note reduction or by an increase in the content of the fruity markers over the short term as well as the long term, it can also decrease the intensity of fruity notes, either by the degradation of these same markers or by the development of an aromatic mask (lactic, smoked/toasted).

The metabolic profile of the wines was modified significantly by the criss-crossed yeast/LAB interactions that are favoured in co-inoculation and that modify the metabolism of wine microorganisms. All of the metabolic activities involved in the synthesis of the aromatic compounds analysed were changed by these interactions, but not in a homogeneous way.

Overall, the metabolites derived from fermentation were affected more by the timing of inoculation than by the varietal compounds, except in the case of the higher alcohols.

Among all of the compounds derived from yeast metabolism, the esters are probably the group that is affected the most by yeast/LAB interactions in co-inoculation. The lack of trends observed for this group of compounds reflects the contradictory results of the previous works (Abrahamse \& Bartowsky, 2011; Knoll et al., 2011; Izquierdo Cañas et al., 2012). However, the changes in fatty acid esters arising from the timing of inoculation, and the corresponding fatty acid composition, are similar (Table 5). They probably reflect the impact of the LAB on lipid metabolism in yeast, especially 
TABLE 5

Trends in the variations of some fatty acid ethyl esters and fatty acid concentrations in wines made by yeast/LAB co-inoculation compared to wines made by sequential inoculation.

\begin{tabular}{cccccc}
\hline Compounds/ wine & M1 & M2 & M3 & M4 & + \\
\hline Ethyl hexanoate & na & - & + & + & + \\
Hexanoic acid & na & - & + & + & - \\
\hline Ethyl octanoate & na & - & + & + & - \\
Octanoic acid & na & - & + & + & - \\
\hline Ethyl decanoate & na & - & + & + \\
Decanoic acid & na & - & + \\
\hline
\end{tabular}

$+:$ increase in concentration in co-inoculation compared to the sequential method; -: decrease in concentration in co-inoculation compared to the sequential method; na: data not available.

since the metabolism of the LAB fatty acid esters might be linked to glycerides and not simple to fatty acid metabolism (Antalick et al., 2012). Changes in the composition of the esters (higher alcohol acetates, fatty acid esters) were actually observed in the wines in which co-inoculation led to sequential MLF (M1 and M5). This probably indicates the simple fact that, when wines are inoculated with LAB during $\mathrm{AF}$, it has an impact on yeast metabolism, even if MLF is carried out after AF. LAB can modify yeast metabolism, either by directly altering the metabolites produced by yeast, or through an impact on the expression of yeast genes involved in the biosynthesis of aroma compounds (Rossouw et al., 2012). The similarity of the influence of the timing of inoculation on fatty acid ester contents and fatty acid contents could reflect the impact of LAB inoculation on the yeast transcriptome, as was observed in a recent study (Rossouw et al., 2012). The competition between yeast and LAB to assimilate the nutrients could also be a potential source of variation in the metabolite levels.

Direct bacterial alterations of yeast metabolites could be responsible for the trend of increases in lactone content in the co-inoculation technique compared to the sequential technique. Wanikawa et al. (2000) used a whisky-making process to demonstrate that interactions between yeast and LAB led to the production of lactones. In the wine-making process, these complex interactions between yeast and LAB might be possible in the co-inoculation technique.

It is also possible that yeast might have an impact on LAB metabolism in the co-inoculation technique. In fact, the diacetyl content was modified strongly by the timing of inoculation, whereas this metabolite is mostly derived from bacterial citric acid catabolism. Increases in the levels of diacetyl and compounds derived from its reduction were observed in the three wines in which co-inoculation led to simultaneous fermentation (wines M2, M3, M4). Simultaneous fermentation imparts a stronger reductive power to the medium, which is supposed to decrease the diacetyl concentration by reducing it to acetoin and butan2-3-diols. On the contrary, the simultaneous increase in the levels of diacetyl and compounds derived from its reduction probably reflects a modification in the bacterial citric acid metabolism by yeast in the co-inoculation technique.

\section{CONCLUSIONS}

This work has demonstrated the impact of the timing of inoculation with $\mathrm{LAB}$ on the metabolic profile of wines made on a winery scale. It was clearly shown that these metabolic changes have an impact on the aromatic profile of the wines. In particular, the lactic and fruity notes were changed but, contrary to empirical ideas, co-inoculation can increase or decrease the intensity of these descriptors, either by the production and degradation of metabolites or by the development of an aromatic mask over the short and long term. This lack of a clear trend reflects the complexity of criss-crossed yeast/LAB interactions occurring in coinoculation, irrespective of the timing of MLF. Although some metabolic trends were highlighted, the overall lack of consensus shows that the metabolic and aromatic changes that occur with co-inoculation depend strongly on the yeast and LAB strains, as well as on the composition of the must. The study of yeast/LAB interactions and certain bacterial metabolisms that are not yet well known could be used to identify some yeast/LAB combinations that are efficient for preserving or enhancing the aromatic expression of wines.

\section{LITERATURE CITED}

Abrahamse, C.E. \& Bartowsky, E.J., 2012. Timing of malolactic fermentation inoculation in Shiraz grape must and wine: Influence on chemical composition. World J. Microb. Biot. 28, 255-265.

Alexandre, H., Costello, P.J., Remize, F., Guzzo, J. \& Guilloux-Benatier, M., 2004. Saccharomyces cerevisiae-Oenococcus oeni interactions in wine: Current knowledge and perspectives. Int. J. Food Microbiol. 93, 141-154.

Anocibar Beloqui, A., Kotseridis, Y. \& Bertrand, A., 1996. Détermination de la teneur en sulfure de diméthyle dans quelques vins rouges. J. Int. Sci. Vigne Vin. 30, 167-170.

Antalick, G., 2010. Biochemical and sensorial modifications of the fruity aroma of red wines during malolactic fermentation: Specific role of esters. Thesis no. 1792, University of Bordeaux II. http://www.theses. fr/2010BOR21792

Antalick, G., Perello, M.C. \& De Revel, G., 2010. Development, validation and application of a specific method for the quantitative determination of wine esters by headspace-solid-phase microextraction-gas chromatographymass spectrometry. Food Chem. 121, 1236-1245.

Antalick, G., Perello, M.C. \& De Revel, G., 2012. Characterization of fruity aroma modifications in red wines during malolactic fermentation. J. Agric. Food Chem. 60, 12371-12383. 
Bertrand, A., 1981. Formation des substances volatiles au cours de la fermentation alcoolique; incidence sur la qualité du vin. Colloque de la Société Française de Microbiologie, Reims.

Curtin, C.D., Bellon, J.R., Henschke, P.A., Godden, P. \& De Barros Lopes, M., 2007. Genetic diversity of Dekkera bruxellensis yeasts isolated from Australian wineries. FEMS Yeast Res. 7, 471-481.

De Revel, G., 1992. Le diacetyl, les composes dicarbonylés et leurs produits de réactions dans les vins. Thesis 190, University Bordeaux II, Bordeaux, France.

De Revel, G., Pipris-Nicolau, L., Barbe, J.C \& Bertrand, A., 2000. The detection of $\alpha$-dicarbonyl compounds in wine by formation of quinoxaline derivates. J. Sci. Food Agr. 80, 102-108.

Diaz-Maroto, M.C., Schneider, R. \& Baumes, R., 2005. Formation pathways of ethyl esters of branched short-chain fatty acids during wine aging. J. Agric. Food Chem. 53, 3503-3509.

Edwards, C.G., Reynolds, A.G., Rodriguez, A.V., Semon, M.J. \& Mills, J.M., 1999. Implication of acetic acid in the induction of slow/stuck grape juice fermentations and inhibition of yeast by Lactobacillus sp. Am. J. Enol. Vitic. 50, 204-210

Escudero, A., Campo, E., Farina, L., Cacho, J. \& Ferreira, V., 2007. Analytical characterization of the aroma of five premium red wines. Insights into the role of odor families and the concept of fruitiness of wines. J. Agric. Food Chem. 55(11), 4501-4510.

Gerbaux, V., Briffox, C., Dumont, A. \& Krieger, S., 2009. Influence of inoculation with malolactic bacteria on volatile phenols. Am. J. Enol. Vitic. 60, 233-235.

Izquierdo Cañas, P., Pérez-Martín, F., García Romero, E., Seseña Prieto, S. \& Palop Herreros, M.L.L., 2012. Influence of inoculation time of an autochthonous selected malolactic bacterium on volatile and sensory profile of Tempranillo and Merlot wines. Int. J. Food Microbiol. 156, 245-254.

Jussier, D., Dube Morneau, A. \& Mira de Orduna, R., 2006. Effect of simultaneous inoculation with yeast and bacteria on fermentation kinetics and key wine parameters of cool-climate Chardonnay. Appl. Environ. Microbiol. 72, 221-227.

Knoll, C., Fritsch, S., Schnell, S., Grossmann, M., Krieger-Weber, S., Du Toit, M. \& Rauhut, D., 2012. Impact of different malolactic fermentation inoculation scenarios on Riesling wine aroma. World J. Microb. Biot. 28, 1143-1153.

Krieger, S. \& Arnink, K., 2003. Malolactic fermentation - A review of recent research on timing of inoculation and possible yeast-bacteria combinations. 32nd Annual New York Wine Industry Workshop, New York.

Martin, N. \& de Revel, G., 1999. Sensory evaluation: scientific bases and oenological applications. J. Int. Sci. Vigne Vin, Special Issue. 81-93.
Massera, M., Soria, A., Catania, C., Krieger, S. \& Combina, M., 2009. Simultaneous inoculation of Malbec (Vitis vinifera) musts with yeast and bacteria: Effects on fermentation performance, sensory and sanitary attributes of wines. Food Technol. and Biotech. 47, 192-201.

Mendoza, L.M., Merín, M.G., Morata, V.I. \& Farías, M.E., 2011 Characterization of wines produced by mixed culture of authochthonous yeasts and Oenococcus oeni from the northwest region of Argentina. J. Ind. Microbiol. Biotechnol. 38, 1777-1785.

Nehme, N., Mathieu, F. \& Taillandier, P., 2010. Impact of the co-culture of Saccharomyces cerevisiae-Oenococcus oeni on malolactic fermentation performance and partial characterization of a yeast-derived inhibitory peptidic fraction. Food Microbiol. 27, 150-157.

Nielsen, J.C., Prahl, C. \& Lonvaud, A., 1996. Malolactic fermentation in wine by direct inoculation with freeze-dried Leuconostoc oenos cultures. Am. J. Enol. Vitic. 47, 42-48.

OIV-MA-AS315-02A. International methods of analysis of wines and must OIV (International Organisation of Vine and Wine). http://www.oiv.int/oiv/ info/enmethodesinternationalesvin?lang=en

Pan, W., Jussier, D., Terrade, N., Yada, R.Y. \& Mira de Orduna, R., 2011 Kinetics of sugars, organic acids and acetaldehyde during simultaneous yeast-bacterial fermentations of white wine at different $\mathrm{pH}$ values. Food Res. Int. 44, 660-666.

Pineau, B., Barbe, J.C., Van Leeuwen, C. \& Dubourdieu, D., 2009. Examples of perceptive interactions involved in specific "red-and-blackberry" aromas in red Wines. J. Agric. Food Chem. 57(9), 3702-3708.

Rasmussen, J.E., Schultz, E., Snyder, R.E, Jones, R.S. \& Smith, C.R., 1995. Acetic acid as a causative agent in producing stuck fermentations. Am. J. Enol. Vitic. 46, 278-280.

Ribéreau-Gayon, P., Dubourdieu, D., Donèche, B. \& Lonvaud, A., 2000 ( $5^{\text {th }}$ ed). Handbook of Enology, vol 2. Microbiology of wine and vinification. Wiley and Sons, Chichester.

Rosi, I., Fia, G. \& Canuti, V., 2006. Influence of different pH values and inoculation time on the growth and malolactic activity of a strain of Oenococcus oeni. Aust. J. Grape Wine R. 9, 194-199.

Rossouw, D., Du Toit, M. \& Bauer, F., 2012. The impact of co-inoculation with Oenococcus oeni on the transcriptome of Saccharomyces cerevisiae and on the flavour-active metabolite profiles during fermentation in synthetic must. Food Microbiol. 29, 121-131.

Sensory Analysis - Apparatus - Wine-Tasting Glass: ISO 3591. In Analyse Sensorielle. AFNOR: Paris, France, 1977.

Wanikawa, A., Hosoi, K. \& Kato, T., 2000. Conversion of unsaturated fatty acids to precursors of gamma-lactones by lactic acid bacteria during the production of malt whisky. J. Am. Soc. Brew. Chem. 58, 51-56.

Zapparoli, G., Tosi, E., Azzolini, M., Vagnoli, P. \& Krieger, S., 2009. Bacterial inoculation strategies for the achievement of malolactic fermentation in high-alcohol wines. S. Afr. J. Enol. Vitic. 30, 49-55. 